\title{
Einleitung zum Forum: „Evaluation der Friedens- und Konfliktforschung durch den Wissenschaftsrat"“
}

\author{
Hartwig Hummel · Solveig Richter · Alexander Spencer
}

Online publiziert: 27. Mai 2020

(C) Der/die Autor(en) 2020

Die Evaluation der deutschen Friedens- und Konfliktforschung durch den Wissenschaftsrat hat in den letzten drei Jahren nicht nur die wissenschaftliche Gemeinschaft intensiv in einen Austauschprozess mit sich und dem Wissenschaftsrat gebracht. Der Bericht ist auch richtungsweisend für die weitere Ausrichtung von Förderprogrammen, Forschungsprojekten und strukturbildenden Lehrinitiativen. Daher haben wir als Herausgeber*innen in einem gesonderten Forum eine Reihe von Autor*innen versammelt, die stellvertretend für die wissenschaftliche Gemeinschaft der Friedensund Konfliktforscher ihre Perspektive auf die Evaluation darstellen.

Kurz noch einmal als Hintergrund: Auf Beschluss des Deutschen Bundestages vom Januar 2017 nahm der Wissenschaftsrat eine Strukturevaluation des Forschungs- und Lehrfeldes der Friedens- und Konfliktforschung sowie im Speziellen auch der Deutsche Stiftung Friedensforschung (DSF) vor. ${ }^{1}$ Im Juli 2019 veröffentlichte der Wissenschaftsrat einen ausführlichen Bericht, der auch online verfügbar ist. Als Einleitung zu den Forumsbeiträgen wollen wir hier einige zentrale Empfehlungen aus der Zusammenfassung des Berichts vorstellen (Wissenschaftsrat 2019a,

\footnotetext{
1 Eine ausführliche Darstellung des Hintergrundes findet sich im Beitrag von Held und Schneckener in diesem Forum.
}

H. Hummel

Heinrich-Heine-Universität Düsseldorf, Düsseldorf, Deutschland

E-Mail: hartwig.hummel@uni-duesseldorf.de

S. Richter

Willy Brandt School of Public Policy, Universität Erfurt, Erfurt, Deutschland

E-Mail: solveig.richter@uni-erfurt.de

\section{A. Spencer $(\bowtie)$}

Otto-von-Guericke-Universität Magdeburg, Magdeburg, Deutschland

E-Mail: alexander.spencer@ovgu.de 
S. 8-12). In einer Bundestagsdebatte am 13. Februar 2020 griffen die Bundestagsparteien den Bericht auf und diskutierten darauf basierend weitere Maßnahmen.

Der Wissenschaftsrat bescheinigt der deutschen Friedens- und Konfliktforschung, ,mit großem Weitblick und der erforderlichen Flexibilität politisch wie gesellschaftlich relevante Themen zu bearbeiten. Die Ergebnisse dieser Forschung werden breit publiziert und stoßen teilweise auch international auf große Aufmerksamkeit" (Wissenschaftsrat 2019b). Der im Sommer 2019 verabschiedete Evaluationsbericht enthält somit nicht nur ein unerwartet großes Lob sondern ist in der Tat auch eine umfassende und lesenswerte Bestandsaufnahme der Friedens- und Konfliktforschung in Deutschland. Er empfiehlt vor allem deren Weiterentwicklung durch konkrete Förderprogramme auf Bundes- und Länderebene (Wissenschaftsrat 2019a).

Die Stimme des Wissenschaftsrats hat ein hohes Gewicht in der deutschen Wissenschaftspolitik. Im Rat arbeiten von den Spitzenorganisationen der Wissenschaft nominierte und vom Bundespräsidenten berufene Wissenschaftlerinnen und Wissenschaftler, ausgewählte Persönlichkeiten des öffentlichen Lebens und Vertreter der Fachressorts des Bundes und der Länder zusammen. Der Rat spricht offiziell nur Empfehlungen aus. Diese haben jedoch eine große politische Bindewirkung und werden in der Regel auch weitgehend von den politisch verantwortlichen Institutionen umgesetzt (Jens 2006, S. 131).

Die zentralen Empfehlungen des Wissenschaftsrats lassen sich wie folgt zusammenfassen:

1. Der Wissenschaftsrat empfiehlt den Ländern, zur „Stärkung der Vernetzung und Profilbildung " regionale Kooperationen und Cluster zu fördern. Naheliegend wäre das wohl für die Regionen Hamburg, Berlin, Hessen und eventuell auch für NRW.

2. Der Wissenschaftsrat rät dazu, bei Fördermaßnahmen die Interdisziplinarität der Friedens- und Konfliktforschung über die bisherigen sozialwissenschaftlichen Kerndisziplinen hinaus zu stärken.

3. Die konkreteste und angesichts der prekären Lage auch dringendste Empfehlung betrifft die naturwissenschaftliche und technische Friedens- und Konfliktforschung. Sie soll ,,an mindestens zwei Standorten, an denen sie bereits institutionell verankert ist, durch Einrichtung einer ausreichenden Anzahl dauerhafter Leitungsstellen (in der Regel zwei je Standort) und befristeter Qualifikationsstellen" ausgebaut werden.

4. Der Wissenschaftsrat wünscht sich eine stärkere Internationalisierung des Forschungsfeldes. Hier sind tatsächlich gerade in jüngster Zeit bereits erhebliche Fortschritte gemacht worden. So spiegelt etwa auch die Öffnung der ZeFKo für englischsprachige Beiträge diesen Trend wider, was allerdings in der Evaluation noch nicht berücksichtigt werden konnte.

5. Last but not least sollen die Fachministerien des Bundes und der Länder die einschlägige Forschungsförderung ausbauen und dabei auch die Finanzierung der Deutschen Stiftung Friedensforschung (DSF) rasch verbessern. Der letztere Punkt wird erfreulicherweise zum Teil bereits umgesetzt, wie Schneckener und Held in ihrem Beitrag berichten können.

Diese Empfehlungen haben natürlich ganz unterschiedliche Hintergründe und Auswirkungen, die wir als ZeFKo-Herausgeber*innen gern aufgreifen. Wir freuen 
uns sehr, dass wir auf unsere Anfragen an die einschlägigen Stellen ein so positives Echo erhalten haben, und möchten uns dafür bei allen Autor*innen ganz herzlich bedanken.

Ihre Erfahrungen als (ehemalige) Vorsitzende der Arbeitsgemeinschaft für Friedens- und Konfliktforschung (AFK), des wichtigsten Netzwerks der Friedens- und Konfliktforschung im deutschsprachigen Raum, nehmen Bettina Engels und Conrad Schetter als Grundlage ihrer Stellungnahme. Seine Erfahrungen beim Auf- und Ausbau einschlägiger Masterprogramme vor dem Hintergrund der Profilierung des Forschungsfeldes lässt Thorsten Bonacker von der Philipps-Universität Marburg in seine kritische Reflektion einfließen. Aus dem Kreis der Forschungsinstitute erhielten wir auf unsere Anfrage einen Beitrag vom IFSH von Holger Niemann und Ursula Schröder. Für die naturwissenschaftliche Friedens- und Konfliktforschung, die gegenwärtig besonders der Unterstützung bedarf und deren Förderung im Gutachten als vorrangig empfohlen wurde, nehmen vier Vertreter in einem gemeinsamen Beitrag Stellung (Christian Reuter, Jürgen Altmann, Malte Göttsche, Mirko Himmel). Dort betonen sie unter anderem, wie notwendig eine enge Vernetzung von natur- und sozialwissenschaftlicher Friedens- und Konfliktforschung ist. Die Erleichterung und Freude über eine verstärkte finanzielle Unterstützung kommt in der Stellungnahme der DSF von Ulrich Schneckener und Thomas Held zum Ausdruck.

Schließlich kommt auch Kai Gehring (Bündnis 90/Die Grünen), selbst Sozialwissenschaftler und einer der aktivsten Bundestagsabgeordneten in Sachen Friedensund Konfliktforschung, zusammen mit seinem wissenschaftliche Mitarbeiter Henrik Rubner in diesem Forum zu Wort. Er brachte für seine Fraktion im Oktober 2019 den Antrag „Ein Kompass in unruhigen Zeiten - Friedensforschung als Grundlage der Politik stärken“ in den Bundestag ein und vertrat ihn am 13. Februar 2020 in der Plenardebatte (Deutscher Bundestag 2020). Mit Ausnahme der AfD sprachen sich in dieser Debatte erfreulicherweise alle Fraktionen für die Förderung der Friedensund Konfliktforschung aus.

Wir hoffen, dass wir mit diesem Forum zur kritischen Selbstreflexion unserer Disziplin beitragen und den Ball des Wissenschaftsrates aufgreifen können. Politische Initiativen zur Förderung der Friedens- und Konfliktforschung sind im Übrigen nicht nur im Bund, sondern auch auf Länderebene und seitens der Hochschulen im Gange. Wir wollen in diesem Forum und auch weiterhin in unserem Heft die Gelegenheit bieten, diese Prozesse kritisch zu begleiten. Um den Schwung des Evaluationsberichtes auch weiterhin in die wissenschaftliche Gemeinschaft zu tragen, sind gerade auch Repliken, die sich mit dem Prozess, den Ergebnissen und den Konsequenzen auseinandersetzen, herzlich willkommen.

Funding Open Access funding provided by Projekt DEAL.

Open Access Dieser Artikel wird unter der Creative Commons Namensnennung 4.0 International Lizenz veröffentlicht, welche die Nutzung, Vervielfältigung, Bearbeitung, Verbreitung und Wiedergabe in jeglichem Medium und Format erlaubt, sofern Sie den/die ursprünglichen Autor(en) und die Quelle ord- 
nungsgemäß nennen, einen Link zur Creative Commons Lizenz beifügen und angeben, ob Änderungen vorgenommen wurden.

Die in diesem Artikel enthaltenen Bilder und sonstiges Drittmaterial unterliegen ebenfalls der genannten Creative Commons Lizenz, sofern sich aus der Abbildungslegende nichts anderes ergibt. Sofern das betreffende Material nicht unter der genannten Creative Commons Lizenz steht und die betreffende Handlung nicht nach gesetzlichen Vorschriften erlaubt ist, ist für die oben aufgeführten Weiterverwendungen des Materials die Einwilligung des jeweiligen Rechteinhabers einzuholen.

Weitere Details zur Lizenz entnehmen Sie bitte der Lizenzinformation auf http://creativecommons.org/ licenses/by/4.0/deed.de.

\section{Literatur}

Deutscher Bundestag. 2020. Beratung des Antrags der Abgeordneten Kai Gehring, Ottmar von Holtz, Omid Nouripour, weiterer Abgeordneter und der Fraktion BÜNDNIS 90/DIE GRÜNEN ,Ein Kompass in unruhigen Zeiten - Friedensforschung als Grundlage der Politik stärken“ Drucksache 19/14111. In Deutscher Bundestag. Stenografischer Bericht. 146. Sitzung, 13. Februar 2020. Plenarprotokoll 19/146. https://dipbt.bundestag.de/dip21/btp/19/19146.pdf\#P.18349. Zugegriffen: 22. Febr. 2020

Jens, Uwe. 2006. Politikberatung und demokratische Legitimität. In Handbuch Politikberatung, Hrsg. Svenja Falk, Dieter Rehfeld, Andrea Römmele, und Martin Thunert, 126-137. Wiesbaden: VS.

Wissenschaftsrat. 2019a. Empfehlungen zur Weiterentwicklung der Friedens- und Konfliktforschung. Drs. 7827-19. Gießen: Wissenschaftsrat. https://www.wissenschaftsrat.de/download/2019/7827-19. pdf?_blob=publicationFile\&v=7. Zugegriffen: 22. Febr. 2020.

Wissenschaftsrat. 2019b. Förderprogramme empfohlen. Wissenschaftsrat plädiert für weitere Stärkung der Friedens- und Konfliktforschung. Wissenschaftsrat Pressemitteilung vom 15.07.2019. https://www. wissenschaftsrat.de/SharedDocs/Pressemitteilungen/DE/PM_2019/pm_1619.html. Zugegriffen: 22. Febr. 2020. 